\title{
USE OF ELASTIC WAVE REFLECTION MECHANISM FOR DETERMINING CON- TACT STRESSES IN SHROUDED TOOLS
}

\author{
M. Mikhovski ${ }^{1 *}$ \\ ${ }^{I}$ V.A. Belyi Metal Polymer Research Institute of National Academy of Sciences of Belarus, 4 Akad. G. Boncheva st., \\ Sofiya, Republic of Belarus. \\ *Corresponding author. E-mail: nntdd@abv.bg ; address for correspondence: ul. Akad. G. Boncheva 4, 000000, Sofiya, \\ Republic of Belarus. Tel.: +35929797120 .
}

The paper presents an ultrasonic method for determining the acting mechanical stresses in the matrix-band contact layer in the shrouded tools for pressing. It also deals with a physical model based on the theory of wave propagation in porous laminated structures. The results of monitoring the shrouded tools are presented.

Key words: shrouded tools, ultrasonic waves, contact stresses.

\section{References}

1. Kortenski Kh., Marinov M., Mikhovski M., Pushev G., Manolova V. Method for determining contact stresses in shrouded tools, inventor's certificate 26905 MPK 601N29/04.

2. Marinov M., Petkanchin L., Pushev G., Kostov G., Mikhovski M., Manolova V.. Device for determining contact stresses in shrouded tools, inventor's certificate 37491 MPK 601N29/04.

3. Trenie, iznashivanie i smazka. Kn.2 (Friction, Wear \& Lubrication. Vol 2). Ed.: I.V. Kragelskii, V.V. Alisin. Moscow, Mashinostrenie Publ., 1979. 358 p. (In Russian).

4. Demkin N.V. Kontaktirovanie scherokhovatykh poverkhnostei (Contact of Rough Surfaces). Moscow, Nauka Publ., 1970, 226 p. (In Russian).

5. Mikhovski M. Investigation of possibility for non-destructive testing the iron-based powder materials. Ph. D. diss., Taganrog, 1973.

6. Brekhovskikh L.M. Volny v sloistykh sredakh (Waves in Stratified Medium). Moscow, AN SSSR Publ., 1957. 343 p. (In Russian). 
УДК 620.179.16:53.082.4

\title{
ИСПОЛЬЗОВАНИЕ МЕХАНИЗМА ОТРАЖЕНИЯ УПРУГИХ ВОЛН ДЛЯ ОПРЕДЕЛЕНИЯ КОНТАКТНЫХ НАПРЯЖЕНИЙ В БАНДАЖИРОВАННЫХ ИНСТРУМЕНТАХ
}

\author{
М. Миховски ${ }^{1 *}$ \\ ${ }^{1}$ Государственное научное учреждение «Институт механики металлополимерных систем им. В. А. Белого \\ Начиональной академии наук Беларуси», София, ул. акад. Г. Бончева, бл. 4. Болгария \\ *Ответственный автор. Электронная почта: nntdd@abv.bg; адрес для переписки: ул. акад. Г. Бончева, 4, София, \\ Республика Болгария. Телефон: +35929797120.
}

В работе представлены ультразвуковой метод определения действующих механических напряжений в контактном слое матрица-бандаж в бандажированных инструментах для прессования и физическая модель метода, разработанного на основе теории распространения упругих волн в слоистых структурах. Приведены результаты контроля бандажированных инструментов.

Ключевые слова: бандажированные инструменты, упругие волны, контактные напряжения.

\section{1. Введение}

Надежность инструмента для обработки металлов и сплавов пластическим деформированием зависит от изнашивания и разрушения пуансонов и матриц. Износостойкость инструмента определяется использованной сталью, технологической смазкой, шероховатостью поверхностей и температурой в рабочей зоне. Разрушение инструмента связано прежде всего с величиной и характером распределения механических напряжений. В бандажированных инструментах для пластической деформации разрушение пуансона наблюдается редко. Обычно в матрицах возникают трещины, ориентированные в продольном и в поперечном направлениях. Путем применения бандажирования матрицы можно оптимизировать уровень механических напряжений и обеспечить долговечную и эффективную работу инструмента.

Существуют различные методы определения механических напряжений в контактном слое между матрицей и бандажом. Большое практическое применение имеет ультразвуковой метод исследования контактного взаимодействия. В этом методе используется зависимость параметров (прежде всего затухания и скорости) ультразвуковой волны, которая отражается от контактного слоя, от акустических характеристик этого слоя. В [1, 2] описаны ультразвуковой метод и аппаратура для неразрушающего контроля действующих контактных напряжений в инструментах, предназначенных для холодного деформирования. Информативным параметром является амплитуда сигнала, отраженного от контактного слоя.

\section{2. Акустическая модель метода}

Акустическая модель для описания явлений, происходящих в контактной зоне при нагружении плоскопараллельных пластин, разработана с учетом трибологических явлений $[3,4]$, особенностей распространения упругих волн в пористых материалах [5] и теории распространения упругих волн в слоистых средах [6].

На плоскую границу двух однородных изотропных сред с одинаковыми акустическими свойствами падает продольная упругая волна. Шероховатость поверхностей контактирующих сред приводит к образованию контактной зоны, размер которой

Mikhovski M. / Use of elastic wave reflection mechanism for determining

contact stresses in shrouded tools 
значительно меньше размеров соприкасающихся сред и длины волны. Из-за наличия конконтактной зоны происходит частичное отражение и прохождение упругой волны во вторую среду. Амплитуды падающих и отраженных упругих волн обозначены соответственно $A$ и $A_{0}$, причем $A_{0}=A R, R-$ коэффициент отражения слоя.

При исследовании контактных явлений в трех пластинах, подвергнутых механическому деформированию, информациоными сигналами явлются:

$$
\begin{gathered}
A_{0}=A R_{1,2} ; \\
A_{00}=A D_{1,2} D_{2,1} R_{2,3},
\end{gathered}
$$

где $R_{i, k}$ - коэффициент отражения от контактного слоя между пластинами $i$ и $k$, $D_{1,2}, D_{2,1}$ - коэффициенты прохождения ультразвуковых волн на границах 1 и 2,2 и 1 пластин. При отсуствии контактного слоя считается, что $R=1$ и $A=A_{0}$. $A_{00}$ - амплитуда отраженного сигнала на границе $2-3, R_{2,3}$ - коэффициент отражения на границе 2-3.

Согласно [6], для случая нормального падения упругой волны на слой зависимость амплитудного коэффициента отражения $R$ от слоя описывается следующим выражением:

$$
R^{2}=\frac{\left(m^{2}-1\right)^{2}}{4 m^{2} \operatorname{ctg} 2 \pi \delta_{x} f / c_{x}+\left(m^{2}+1\right)^{2}},
$$

где $m=z_{\mathrm{x}} / z ; z_{\mathrm{x}}=\rho_{\mathrm{x}} c_{\mathrm{x}} ; z=\rho c$ - акустические сопротивления; $\rho_{\mathrm{x}}, \rho, c_{\mathrm{x}}, c$-плотности и скорости распространения упругих продольных волн в контактном слое и в прилегающих средах; $f$ - частота упругой волны; $\delta_{\mathrm{x}}$ - толщина контактного слоя.

Слой между средами 1 и 2 рассматривается как пористая среда, состоящая из статистической совокупности упругих частиц. Если со стороны прилегающих сред на граничный слой действует равномерно распределенная сила $P$, изменяющаяся от нуля до заданного значения $P_{k}$, то происходит изменение акустических характеристик слоя, равномерное по всей его протяженности. Величина силы $P_{k}$ всегда может быть выбрана так, что акустические параметры прилегающих сред будут оставаться постоянными при действии силы $P<P_{k}$. Передача давления через граничный слой реализуется через множество единичных контактов дискретных частиц слоя, причем давление в этих точках контакта значительно превышает величину среднего давления на контактный слой. Согласно современным представлениям о деформировании нагруженного контактного слоя в условиях трения покоя $[3,4]$ его уплотнение проходит в три стадии: уплотнение из-за скольжения и деформирования крупных неровностей, уплотнение за счет деформирования приконтактных областей и уплотнение при деформировании больших объемов слоя. В слое действуют контактные напряжения $\sigma=P / s$, где $s-$ площадь контактирующих пластин.

Слой характеризуется толщиной $(\delta)$, пористостью $(p)$, плотностью ( $\rho)$ и скоростью распространения продольных упругих волн $(c)$. Индексы 0 и $x$ соответствуют характеристикам ненагруженного слоя и для слоя с приложенной нагрузкой $P$ соответственно. Для пористых сред зависимости между акустическими характеристиками можно выразить следующим образом:

$$
\begin{array}{ll}
c_{x}=c /\left(1-a p_{x}\right), & c_{0}=c /\left(1-a p_{0}\right), \\
\rho_{x}=\rho /\left(1-p_{x}\right), & \rho_{0}=\rho /\left(1-p_{0}\right),
\end{array}
$$

Mikhovski M. / Use of elastic wave reflection mechanism for determining contact stresses in shrouded tools 
где $a$ - постоянная величина, определяемая экспериментально. Исследование изменения геометрических характеристик контакта двух шероховатых сред в условиях нагружения показывает, что толцина слоя изменяется по закону $[3,4]$ :

$$
\delta_{x}=\delta_{0}[1-F(P)]
$$

Функциональные зависимости $F(P)$ получены экспериментально для случаев контакта двух сред с различной шероховатостью и волнистостью, для сред с различными механическими характеристиками в условиях упругого и пластического деформирования при однократном и многократном нагружении. Следовательно, эта функция известна, если имеются данные о механических характеристиках прилегающих сред, микрогеометрии контактирующих поверхностей и режимах нагружения. Толщина слоя $\delta_{0}$ определяется исходя из соображений $[3,4]$ для различных случаев контактного взаимодействия.

В процессе деформирования контактного слоя изменяются толщина и пористость слоя, а его масса сохраняется. Следовательно, для контактного слоя может быть записано следующее равенство:

$$
\rho_{x} \delta_{x}=\rho_{0} \delta_{0}
$$

После подстановки (3) и (4) в (2) зависимости пористости, плотности и скорости распространения упругих волн в слое от приложенной нагрузки принимают вид:

$$
\begin{array}{ccc}
\rho_{x}=\rho A / B, & p_{x}=1-A / B, & c_{x}=c B / M, \\
A=1-p_{0}, & B=1-F(P), & M=B(1-a)+a A .
\end{array}
$$

Амплитудный коэффициент отражения упругих волн от контактного слоя, с учетом сделанных преобразований, определяется по формуле:

$$
R^{2}=\frac{\left(A^{2}-M^{2}\right)^{2}}{4 A^{2} M^{2} \operatorname{ctg} 2 \pi \delta M / c+\left(A^{2}+M^{2}\right)^{2}}
$$

На рис. 1 представлена часть результатов расчетов изменения коэффициента отражения упругих волн $\Delta R$ для случая пластического деформирования контактных областей в условиях однократного нагружения. Кривые даны для различных частот (3, 6 и 12 МГц) упругих волн, для различных степеней шероховатости контактирующих поверхностей и толщин контактного слоя $\delta$ и пористости при условии, что шероховатость контактирующих поверхностей одинакова.

На рис. 2 представлена расчетная зависимость $\Delta R=F(\sigma)$ для случая однократного нагружения плоскопараллельных пластин из стали 40X и X12M твердостью 40 - 50 HRC. Расчеты сделаны для частоты ультразвукового сигнала 12 МГц, $\delta_{0}=9,4$ мкм и $p_{0}=0,55$. Точками обозначены результаты эксперимента определения $\Delta R$ при нагружении пяти пар пластин. Различие экспериментальных и расчетных данных не превышает 0,2 дБ. 


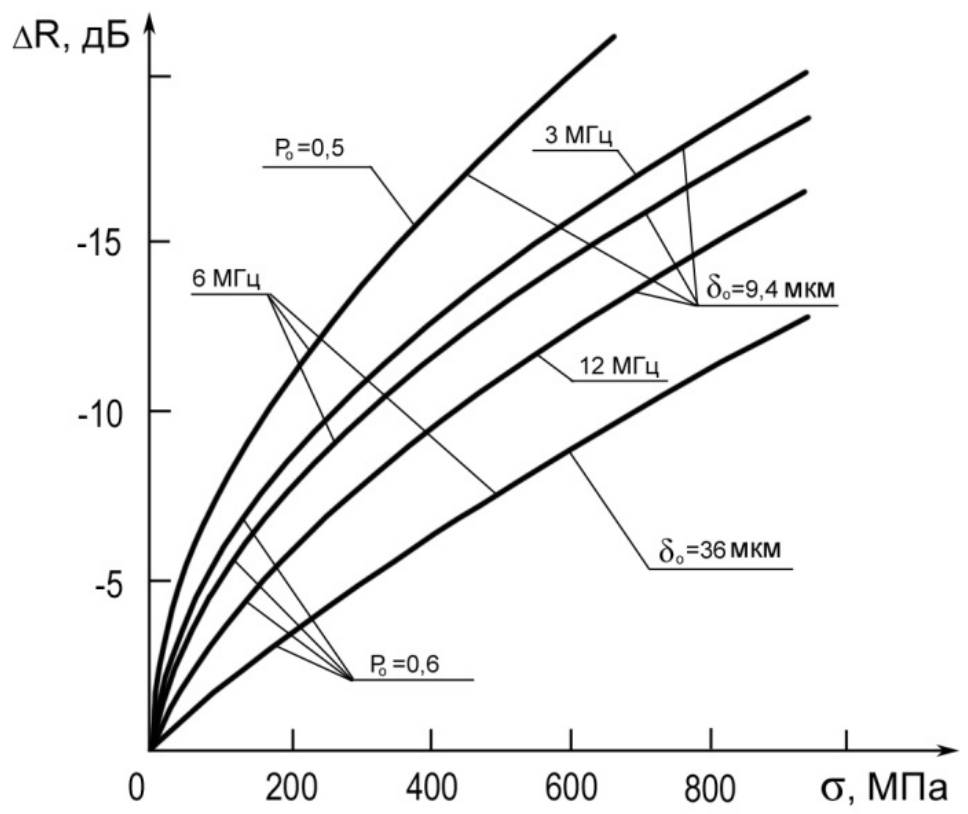

Рис. 1. Изменение коэффициента отражения $\Delta R$ при нагрузке пластин в зависимости от частоты $f$, толщины слоя $\delta$ и пористости $p$

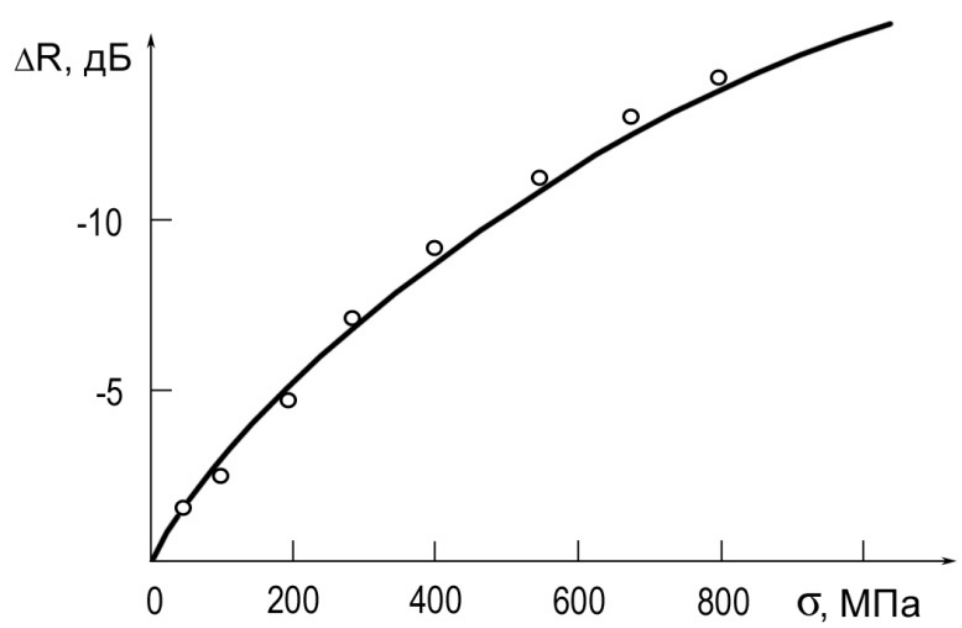

Рис. 2. Калибровочная кривая $\Delta R=F(\sigma)$ для контактного слоя стали Х12М и стали $40 \mathrm{X}$

Анализ полученных результатов показывает хорошее совпадение расчетных и экспериментальных данных, что дает основание использовать принятую акустическую модель в экспериментах, связанных с получением калибровочных зависимостей для определения действующих контактных напряжений в инструментах для холодной объемной деформации.

Исходя из полученых результатов можно рекомендовать оптимизацию выбора рабочих частот с целью увеличения чувствительности ультразвукового неразрушающего метода определения контактных напряжений.

\section{3. Аппаратура. Методика. Результаты}

На рис. 3 представлена блок-схема разработанной специализированной аппаратуры контроля бандажированных инструментов для изготовления болтов. Она включает ультразвуковой дефектоскоп (1) с ультразвуковым преобразователем (2), электродвигатель (3), синхронизатор работы электродвигателя (4) и регистрирующее устройство (5). 
Контролирумый инструмент закрепляется на направляющих в стойке. Аппаратура обеспечивает вращательное движение инструмента и поступательное движение ультразвукового преобразователя. С целю повышения точности измерения параметров отраженного от контактного слоя ультразвукового сигнала реализован щелевой контакт в локальной иммерсионной ванне между преобразователем и обьектом контроля. Специальная схема регистрации обеспечивает измерение амплитуды сигнала с погрешностью не более 0,005 мВ. Управление механизмом измерительного стенда, регистрация измеряемых параметров и определение контактных давлений по заранее вводимым калибровочным зависимостям для контролируемых одно- и двухбандажных инструментов осуществляются с помощью персонального компьютера.

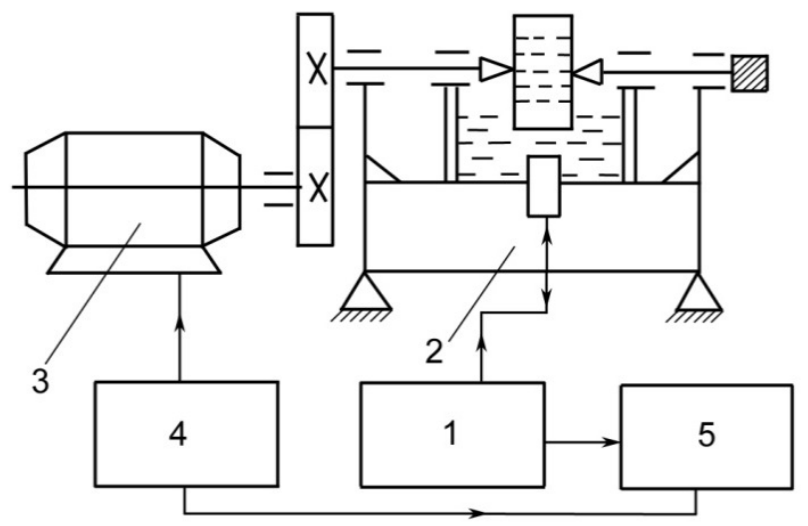

Рис. 3. Блок-схема установки контроля бандажированных инструментов для изготовления болтов:

1 - ультразвуковой дефектоскоп, 2 - ультразвуковой преобразователь, 3 - электродвигатель, 4 - синхронизатор работы электродвигателя, 5 - регистрирующее устройство

На рис. 4 и 5 представлены результаты распределения контактных механических напряжений одно- и двухбандажного инструмента соответствено. Обозначения $\sigma_{b-b}$ и $\sigma_{b-m}$ относятся для распределения напряжений на границах бандаж-бандаж и бандаж-матрица соответствено.

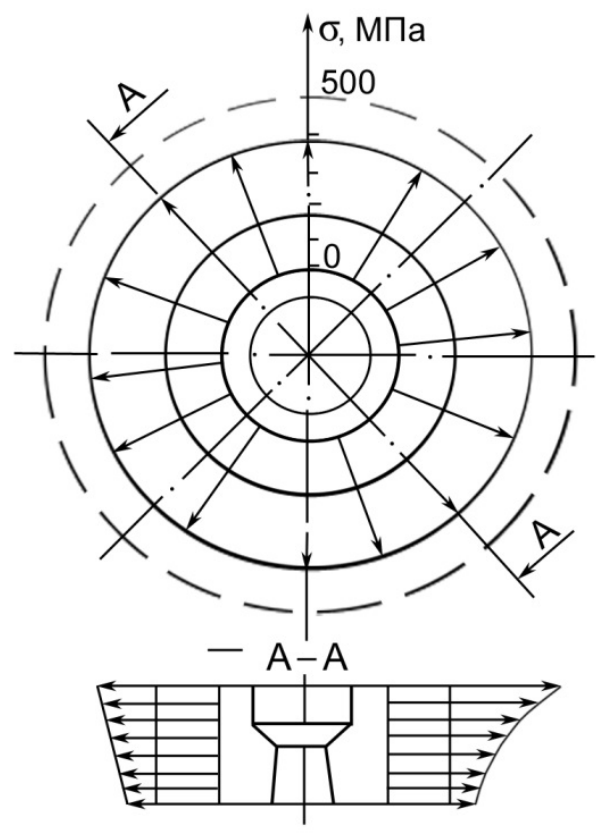

Рис. 4. Распределение контактных механических напряжений в однобандажном инструменте

Mikhovski M. / Use of elastic wave reflection mechanism for determining contact stresses in shrouded tools 


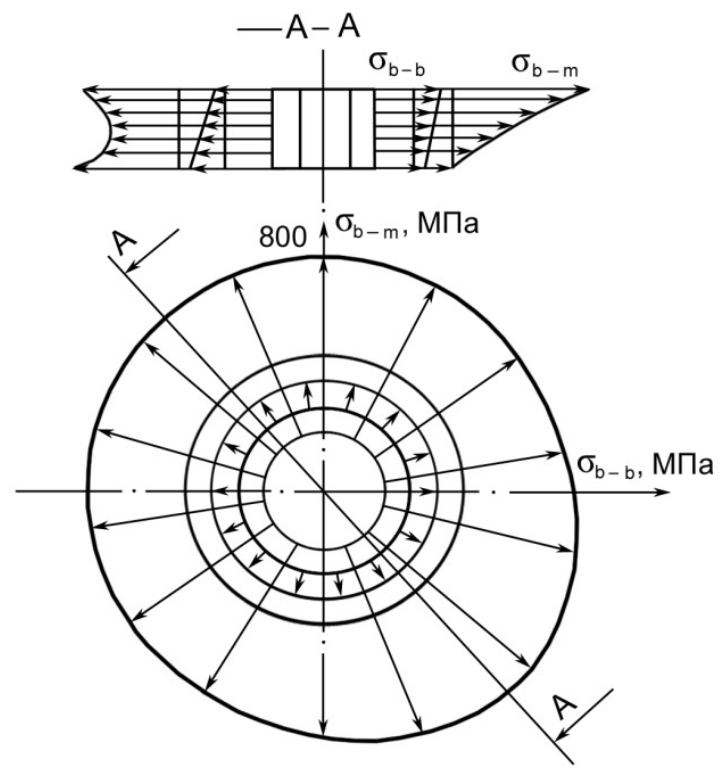

Рис. 5. Распределение контактных механических напряжений в двухбандажном инструменте: $\sigma_{\mathrm{b}-\mathrm{b}}$ - для распределения напряжений на границах бандаж-бандаж, $\sigma_{\mathrm{b}-\mathrm{m}}-$ для распределения напряжений на границах бандаж-матрица

\section{4. Заключение}

Разработанная методика и аппаратура защищены патентами и внедрены на специализированных заводах в Болгарии, России и Венгрии. Ультразвуковая аппаратура была отмечена золотой медалю на ярмарке в Лейпциге.

\section{Литература}

1. Кортенски Х., Маринов М., Миховски М., Пушев Г., Манолова В. Метод для определения контактного напряжения в бандажированных инструментах: а.с. 26905. № МПК $601 \mathrm{~N} 29 / 04$.

2. Маринов М., Петканчин Л., Пушев Г., Костов Г., Миховски М., Манолова В., Устройство для определения контактных напряжений в бандажированных инструментах: a.c. 37491 МПК $601 \mathrm{~N} 29 / 04$.

3. Трение, изнашивание и смазка: Справочник. В 2 кн. / Под ред. И. В. Крагельского, В. В. Алисина. - М.: Машиностроение, 1978 - 1979.

4. Демкин Н. Б. Контактирование шероховатых поверхностей. - М.: Наука, 1970. - 226 с.

5. Миховски М. Исследование возможности неразрушающего контроля порошковых металлургических материалов на основе железа: дис. канд. техн. наук. - Таганрог: ТРТИ, Таганрог, 1973.

6. Бреховских Л. М. Волны в слоистых средах. - М.: АН СССР, 1957. - 343 с. 